\title{
Hypermagnesemia, CTCAE
}

National Cancer Institute

\section{Source}

National Cancer Institute. Hypermagnesemia, CTCAE. NCI Thesaurus. Code C143555.

A disorder characterized by laboratory test results that indicate an elevation in the concentration of magnesium in the blood. 